\title{
AN APPLICATION OF STRESS ENERGY TENSOR TO THE VANISHING THEOREM OF DIFFERENTIAL FORMS
}

\author{
KAIREN CAI \\ Department of Mathematics and Statistics \\ Southern Illinois University at Edwardsville \\ Edwardsville, Illinois 62026-1653 \\ (Received September 29, 1987)
}

\begin{abstract}
The author applies the stress energy of differential forms to study the vanishing theorems of the Liouville type. It is shown that for a large class of underlying manifolds such as the Euclidean n-space, the complex n-space, and the complex hyperbolic space form, if any vector bundle valued p-form with conservative stress energy tensor is of finite norm or slowly divergent norm, then the p-form vanishes. This generalizes the recent results due to Hu and Sealey.
\end{abstract}

KEYWORDS AND PHRASES. Conservation, stress energy tensor, complex hyperbolic space form, vector bundle valued $p$-form.

1980 AMS SUBJECT CLASSIFICATION CODES. $58 \mathrm{Al} 0$.

1. INTRODUCTION.

The classical Liouville theorem states that any bounded harmonic function defined on the entire plane is a constant function. Later many authors generalized the theorem. Yau [1] generalized it to harmonic functions on complete Riemannian manifolds with non-negative Ricci curvature. It was further extended to harmonic maps between Riemannian manifolds by Eells and Sampson [2], Cheng [3], Garbar et al. [4], Hu [5], Xin [6], Sealey [7] and others.

It is well known that if the field equation of a physical system is obtained by means of calculating the derivative of the action functional $I$, we can define a so called stress energy tensor $S$ such that $S$ is conservative at the critical values of I. In particular, the stress at a point of an elastic body is described by a tensor $\left(S_{1 j}\right)$ in the Euclidean space $R^{3}$. The law of conservation of momentum about some origin implies the stress energy tensor $S_{1 j}$ be symmetric and the system be in equilibrium means that $S_{1 j}$ be divergence free. Recently, Baird and Eells [1] proved that if the map between Riemannian manifolds is harmonic, then its stress energy tensor is conservative. This might make the harmonic map a new physical explanation. 
The purpose of the paper is to apply the stress energy tensor of p-form with values in a vector bundle over some kind of Riemannian manifolds to the vanishing theorem of the Liouville type. The main result is the following.

THEOREM 1.1. Let $U^{n}$ be the complex hyperbolic space form and $F$ a Hermitian vector bundle over $U^{n}$. Let $\omega$ be a p-form with values in $F, p<n$. If $w$ is of finite or slowly divergent norm over $U^{n}$ and 1 ts stress energy tensor is conservative, then $\omega=0$.

It must be pointed out that this is a more general vanishing theorem of vector bundle valued p-form. We all know that there are many important maps between Riemannian manifolds such as harmonic, relative harmonic [5], holomorphic [8] and relative affine maps [9] whose differentials possess the free divergent stress energy tensors. Sealey [7] proved that if the norm of a harmonic 1-form with values in a vector bundle over $U^{n}$ is integrable, then the 1-form vanishes. Therefore, Theorem 1 means that under a weaker condition than Sealey's, a stronger conclusion still holds.

In section 2 after introducing some notations, we establish an integral formula of the stress energy tensor and some kind of radial variational formula for p-forms by using the Lie derivative. Besides being applied to the proof of Theorem 1 , these formulas are of interest in its own right. In section 3, we prove Theorem 1.1. by means of estimating the first variation of the norm of p-form motivated by the method in [4]. Finally, we expand our result to the general case where the underlying manifold can be the complex n-space or some kind of conformal flat Riemannian manifolds.

\section{NOTATIONS AND LEMMAS.}

For simplicity, we only state some notations and formulas for the Riemannian vector bundle over real Riemannian manifold, those of Hermitian vector bundle over Kaehler manifold can be written correspondently. Let $F$ be a Riemannian vector bundle over Riemannian manifold $M$. We define an inner product in $\wedge \mathrm{P}_{T_{X}}{ }^{*} \cdot F_{X}$ by setting

$$
\langle\omega, \theta\rangle=\sum_{i_{1}<\ldots<i_{p}}\left\langle\omega\left(e_{i_{1}}, \ldots, e_{1_{p}}\right), \theta\left(e_{1_{1}}, \ldots, e_{1_{p}}\right)\right\rangle
$$

where $w, \theta \in \wedge P_{T_{x}^{*}}^{*} \bullet F_{x},\left\{e_{1}, \ldots, e_{n}\right\}$ is any orthonormal basis of $T_{x} M$. Integrating this pointwise inner product over $M$ gives an inner product in $A^{P}(F)$, which denotes the space of sections of $\wedge^{P_{T}^{*}} M \bullet F$. For a linear connection $\nabla$ on $F$ we define the covariant derivative by

$$
\left(\nabla_{y} \omega\right)\left(x_{1}, \ldots, x_{p}\right)=\nabla_{y} \omega\left(x_{1}, \ldots, x_{p}\right)-\sum_{i=1}^{p} \omega\left(x_{1}, \ldots, \nabla_{y} x_{1}, \ldots, x_{p}\right)
$$

for $Y, X \in A(T M)$. The exterior differential $d: A^{p}(F)+A^{p+1}$ (F) is given by

$$
d \omega\left(x_{1}, \ldots, x_{p+1}\right)
$$




$$
\begin{aligned}
& =\sum_{i=1}^{p+1}(-1)^{i+1} v_{x_{i}}\left(x_{1}, \ldots, \hat{x}_{i}, \ldots, x_{p+1}\right) \\
& +\sum_{i<j}(-1)^{i+j} \ldots\left(\left[x_{i}, x_{j}\right], x_{1}, \ldots, \hat{x}_{i}, \ldots, \hat{x}_{j}, \ldots, x_{p+1}\right)
\end{aligned}
$$

where $x_{i} \equiv A(T M), 1 \cdot i, j \div p+1, \dot{x}_{i}$ means $x_{i}$ is deleted. The codifferential $j: A^{P}(F) \rightarrow A^{P-1}(F), P>1$, is given by

$$
\delta\left(x_{1}, \ldots, x_{p-1}\right)=-\sum_{i=1}^{n} \nabla_{e_{1}} \omega\left(e_{1}, x_{1}, \ldots, x_{p-1}\right) .
$$

The generalized Laplace-Beltrami operator for $p$-form with values in $F$ is defined by $\Delta=\mathrm{d} \delta+\delta \mathrm{d}$. Any $\mathrm{p}$-form $\omega$ with values in $\mathrm{F}$ satisfying the equations $\mathrm{d} \omega=0$, $\delta \omega=0$ is called harmonic. If $M$ is a manifold without boundary and $\omega$ is of compact support, then $\omega$ is harmonic if $\mathrm{f} \omega=0$.

The symmetric square $\omega \cdot \omega$ of $\omega \varepsilon A^{P}(F)$ is a symmetric F-valued 2-tensor defined by

$$
\omega \cdot \nu(X, Y)=\left\langle i_{x} \omega, i_{y} \omega\right\rangle
$$

where $X, Y \in A(T M), i_{X}$ denotes the contraction with $X$. We call

$$
E_{\omega}=\int_{M} e_{\omega} *_{1}
$$

the norm of $\omega$ over $M$ where $e_{\omega}=|\omega|^{2} / 2$. The stress energy tensor of $\omega$ is given by

$$
S_{\omega}=e_{\omega} g-\omega \cdot \omega
$$

in which $g$ is the metric tensor of $M$.

LEMMA 2.1. Suppose $X$ be a vector field with compact support over $M$ and $\omega p$-form with values in a vector bundle, then

$$
\int_{M}\left\langle L_{x} \omega, \omega\right\rangle \star_{1}=\int_{M} \operatorname{div} s_{\omega}(x) *_{1}+\int_{\partial M} \omega \cdot \omega(x, \partial / \partial n) \star_{1}
$$

in which div $\mathrm{S}_{\omega}$ is the divergence of $\mathrm{S}_{\omega}$ and $\partial / \partial \mathrm{n}$ the unit outward normal to $\partial M$. PROOF. Since [6]

we get

$$
\operatorname{div} s_{\omega}(x)=\left\langle i_{x} d \omega, \omega\right\rangle+\left\langle\delta \omega, i_{x} \omega\right\rangle
$$

$$
\begin{aligned}
\left\langle L_{x} \omega, \omega\right\rangle= & \left\langle 1_{x} d \omega+d 1_{x} \omega, \omega\right\rangle \\
& =d 1 v s_{\omega}(x)+\left\langle d 1_{x} \omega, \omega\right\rangle-\left\langle\delta \omega, 1_{x} \omega\right\rangle
\end{aligned}
$$

Integrating and using Green theorem immediately complete the proof. 
Let us consider a special case of the above formula. Assume $\phi: M+N$ be a smooth map from a Riemannian manifold $M$ without boundary to Riemannian manifold N. According to Lemma 1 , we know that

so

$$
\int_{M}\left\langle\mathrm{~L}_{\mathrm{x}} \mathrm{d} \phi, \mathrm{d} \phi\right\rangle \star_{1}=\int_{M}\langle\mathrm{~d} \phi(\mathrm{X}), \delta \mathrm{d} \phi\rangle \star_{1}
$$

$$
\operatorname{siv} s_{\phi}=\left\langle\tau_{\phi}, \mathrm{d} \phi\right\rangle
$$

where $S_{\phi}$ is the stress energy tensor of $\phi$ and $\tau_{\phi}=\delta d \phi$ is the torsion field of $\phi$. Thus, we easily prove

COROLLARY (Baird and Eel1s [10]). The stress energy tensor of a harmonic map from $M$ to $N$ mast be conservative. Conversely, if $\phi$ is a differential submersion and its differential $d \phi$ is of conservative stress energy tensor, then $\phi$ is harmonic.

LEMMA 2.2. Let $U^{n}(\rho)$ be an open disc with radius $\rho$ in the complex n-space $C^{n}$ and $\omega$ be an F-valued $p$-form over $U^{n}(\rho)$. Suppose $\omega_{t}$ be an 1-parameter family of p-forms such that

$$
\omega_{t}\left(z_{1}, \ldots, z_{p}\right)=t^{p}\left(\phi_{t}^{*} \omega_{t z}\right)\left(z_{1}, \ldots, z_{p}\right)
$$

where $z \in U_{*}^{n}(\rho), 0<t|z|<p,\left\{z_{1}, \ldots, z_{p}\right\}$ are the constant vector fields over $U^{n}(\rho)$, and $\phi_{t}^{*}$ is induced from parallel transport in $F$ along the straight line from tz to $z$. Then we have

$$
\left.\frac{d}{d t} \omega_{t}\right|_{t=1}=L_{r \partial / \partial r^{\omega}}
$$

PROOF. This depends largely on a direct computation. One can easily see that at $t=1$

$$
\frac{d}{d t}\left(\phi_{t}^{*} \omega_{t z}\right)\left(z_{1}, \ldots, z_{p}\right)=\nabla_{r \partial / \partial r} \omega\left(z_{1}, \ldots, z_{p}\right)
$$

Therefore at $t=1$

$$
\frac{d}{d t} \omega_{t}\left(z_{1}, \ldots, z_{p}\right)=\nabla_{r} \partial / \partial r\left(z_{1}, \ldots, z_{p}\right)+p \omega\left(z_{1}, \ldots, z_{p}\right)
$$

On the other hand, we may assume $z_{i}=\partial / \partial z_{i}$ or $\partial / \partial \bar{z}_{i}$. Thus, $\left[z_{i}, z_{j}\right]=0$ and $\left[r \partial / \partial r, z_{1}\right]=-z_{1}$ It follows that

$$
\begin{aligned}
& \left(L_{r \partial / \partial r} \omega\right)\left(z_{1}, \ldots, z_{p}\right)=\left(i_{r \partial / \partial r} d \omega+d_{r \partial / \partial r} \omega\right)\left(z_{1}, \ldots, z_{p}\right) \\
& =d \omega\left(r \partial / \partial r, z_{1}, \ldots, z_{p}\right)+\sum_{i=1}^{p}(-1)^{1+1} \nabla_{z_{i}} \omega\left(r \partial / \partial r, z_{1}, \ldots, \hat{z}_{i}, \ldots, z_{p}\right) \\
& =\nabla_{r \partial / \partial r} \omega\left(z_{1}, \ldots, z_{p}\right)+p \omega\left(z_{1}, \ldots, z_{p}\right) .
\end{aligned}
$$


The proof is completed.

From lemmas $2.1,2.2$ we immediate $1 y$ obtain

LEMMA 2.3. If $\omega$ is an F-valued p-form with conservative stress energy tensor, then $d\left\|\omega_{t}\right\|_{p}^{2} / d t>0$ at $t=1$.

Finally, we give the following.

DEFINITION. Let $F$ be a Hermitian vector bundle over $U^{n}$ and $\omega$ be a $p$-form with values in $F$. The norm of $w$ over $U^{n}$ called slowly divergent if

$$
\int_{U} e_{\omega} e_{1}=\infty \quad \text { and } \quad \int_{0}^{1} \frac{f(r)}{1-r} d r=\infty
$$

hold for any positive continuous function $f(r)$ in $(0,1)$ (for example $f(r)=1 / \log (1-r)$ ), we have

$$
\int_{U} \mathrm{f}(r) e_{\omega} \star 1<\infty
$$

3. THE PROOF OF THEOREM 1.1 .

After establishing the preceding lemmas, it is not difficult to prove Theorem 1. We represent the complex hyperbolic space form by the open unit disc $\mathrm{U}^{\mathrm{n}}$ in $\mathrm{C}^{\mathrm{n}}$ with the complete Kaehler metric [11]

$$
g=4 \frac{\left(1-\Sigma z_{\alpha} \bar{z}_{\alpha}\right)\left(\sum \mathrm{dz} \alpha_{\alpha} \mathrm{dz}_{\alpha}\right)+\left(\Sigma_{\bar{z}} \bar{d}_{\alpha}\right)\left(\sum_{z_{\alpha}} \mathrm{dz}_{\alpha}\right)}{\left(1-\Sigma_{z_{\alpha} \bar{z}_{\alpha}}\right)^{2}}
$$

It is obvious to see that $T_{z}^{1,0}\left(U^{n}\right)$ and $T_{z}^{0,1}\left(U^{n}\right)$ have respectively the orthonormal bases

$$
\left\{\frac{1-r^{2}}{2} \frac{\partial}{\partial z_{1}}, \frac{\sqrt{1-r^{2}}}{2} \frac{\partial}{\partial z_{2}}, \ldots, \frac{\sqrt{1-r^{2}}}{2} \frac{\partial}{\partial z_{n}}\right.
$$

and

$$
\left\{\frac{1-r^{2}}{2} \frac{\partial}{\partial \bar{z}_{1}}, \frac{\sqrt{1-r^{2}}}{2} \frac{\partial}{\partial \bar{z}_{2}}, \ldots, \frac{\sqrt{1-r^{2}}}{2} \frac{\partial}{\partial \bar{z}_{n}}\right.
$$

For convienence, we may assume that $\omega$ is of degree $(k, h), k+h=p$. Then $\omega$ can be expressed as

$$
\omega=\Sigma \mathrm{f}_{1_{1}} \ldots 1_{k} j_{1} \cdots j_{h} d z_{1} \wedge \cdots \wedge d z_{1_{k}} \wedge d \bar{z}_{j_{1}} \wedge \ldots \wedge d \bar{z}_{j_{h}} \cdot
$$


To begin with we calculate the inner product $\left|\omega_{t}\right|^{2}$ at the point $\left(z_{1}, 0, \ldots, 0\right)$. At that time, the Kaehler metric is reduced to

$$
g=\frac{4}{\left(1-r^{2}\right)^{2}}\left(d z_{1} d \bar{z}_{1}+\left(1-z^{2}\right) \sum_{2}^{n} d z_{\alpha} d \bar{z}_{\alpha}\right)
$$

We can decompose $\omega_{t}$ to $\omega_{t}=\omega_{t}^{1}+\omega_{t}^{2}+\omega_{t}^{3}$ among which $\omega_{t}^{1}$ consists of such p-forms that do not contain the factors $d z_{1}$ and $d \bar{z}_{1}$ those of $\omega_{t}^{2}$ contain factor $\mathrm{d} z_{1}$ or $\mathrm{d} \bar{z}_{1}$ and $\omega_{t}^{3}$ are the remains. Using $(2.13),(3.3)$, we can compute that

$$
\begin{aligned}
& \left|\omega_{t}^{1}\right|^{2}=t^{2 p}\left|\omega^{1}\right|_{t z}^{2} \frac{\left(1-r^{2}\right)^{p}}{\left(1-t^{2} r^{2}\right)^{p}} \\
& \left|\omega_{t}^{2}\right|^{2}=t^{2 p}\left|\omega^{2}\right|_{t z}^{2} \frac{\left(1-r^{2}\right)^{p+1}}{\left(1-t^{2} r^{2}\right)^{p+1}}, \\
& \left|\omega_{t}^{3}\right|^{2}=t^{2 p}\left|\omega^{3}\right|_{t z}^{2} \frac{\left(1-r^{2}\right)^{p+2}}{\left(1-t^{2} r^{2}\right)^{p+2}},
\end{aligned}
$$

Then, since $U^{n}$ is a homogeneous space, the restriction on $z$ can be removed and these expressions hold for all $z \in v^{\mathrm{n}}$. Denote $d V$ and $d S$ be the volume elements of $U^{n}$ and the hypersphere $S_{r}$ in $U^{n}$ respectively. We have $d V=2 d r d S /\left(1-r^{2}\right)$. Now ass uming

$$
A(r, t)=\frac{2}{1-r^{2}} \int_{S_{r}}\left|\omega_{t}\right|^{2} d s
$$

we get

$$
\left\|\omega_{t}\right\|_{0}^{2}=\int_{0}^{\rho} \frac{2}{1-r^{2}} d r \int_{S}\left|\omega_{t}\right|^{2} d s=\int_{0}^{\rho} A(r, t) d r
$$

and

$$
\begin{aligned}
& \frac{d}{d t} \|_{\omega_{t} \|_{\rho}^{2}}=\frac{d}{d t} t^{2 p-2 n} \int_{|z|<t \rho}\left[\left|\omega^{1}\right|^{2} \frac{\left(1-r^{2} / t^{2}\right)^{p}}{\left(1-r^{2}\right)^{p}}\right. \\
& \left.+\left|\omega^{2}\right|^{2} \frac{\left(1-r^{2} / t^{2}\right)^{p+1}}{\left(1-r^{2}\right)^{p+1}}+\left|\omega^{3}\right|^{2} \frac{\left(1-r^{2} / t^{2}\right)^{p+2}}{\left(1-r^{2}\right)^{p+2}}\right] G_{z / t} d z d \bar{z}
\end{aligned}
$$

where

$$
G_{z / t}=i^{n^{2}} \operatorname{det}\left(\frac{4}{\left(1-r^{2}\right)^{2}}\left[\left(1-r^{2} / t^{2}\right) \delta_{\alpha \beta}+\bar{z} \alpha^{z} \beta^{/ t^{2}}\right]\right)=1^{n^{2}} 4^{n} /\left(1-r^{2} / t^{2}\right)^{n+1} \text {. }
$$

Then at $t=1$

$$
\begin{aligned}
& \frac{d}{d t}\left\|\omega_{t}\right\|_{\rho}^{2}=\rho A(\rho, 1)+2(p-n)\|\omega\|_{\rho}^{2}-\int_{|z|<\rho}\left[(n+1-p)\left|\omega^{1}\right|^{2}+\right. \\
& \left.\quad(n-p)\left|\omega^{2}\right|^{2}+(n-p-1)\left|\omega^{3}\right|^{2}\right] \frac{2 r^{2}}{1-r^{2}} d V \\
& <\rho A(\rho, 1)-2(n-p)\left\{_{|z|<\rho}|\omega|^{2}\left(1+\frac{(n-p-1) r^{2}}{(n-p)\left(1-r^{2}\right)}\right) d V\right.
\end{aligned}
$$


Here we assume $p<n-1$ because the case of $p=n-1$ is simpler than the former and can be $t$ reated by the same way. It follows from Lemma 3.1 that

$$
\rho A(\rho, 1)>2(n-\rho) \int_{z \mid<\rho}|\omega|^{2}\left(1+\frac{(n-p-1) r^{2}}{(n-p)\left(1-r^{2}\right)}\right) d V .
$$

Suppose that $\omega \in A^{P}(F)$ do not equal zero identically on $U^{n}$. Thus there exists $\rho_{0}$ such that $0<\rho_{0}<1$ and

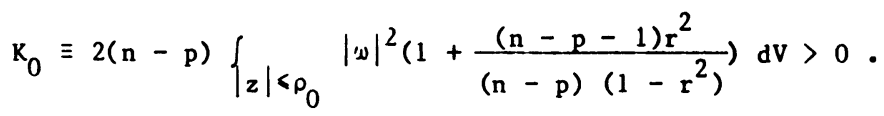

\section{Therefore}

$$
\rho A(\rho, 1)>K_{0}+2(n-p) \int_{\rho_{0}}^{\rho}\left(1+\frac{(n-p-1) r^{2}}{(n-p)\left(1-r^{2}\right)}\right) A(r, 1) d r
$$

According to the method in [7], one can prove that

$$
A(p, 1)>K_{1} /\left(1-r^{2}\right)^{n-p-1}
$$

for some $K_{1}>0$ and all $\rho_{0}<\rho<1$. Then for any positive continuous function $f(r)$ with

$$
\int_{0}^{1} \frac{f(r)}{1-r} d r=\infty
$$

we have

$$
\int_{U^{n}} f(r) e_{\omega} d V>\int_{\rho_{0}}^{1} r f(r) A(r, 1) d r>k_{1} \int_{\rho_{0}}^{1} \frac{f(r)}{1-r^{2}} d r=\infty
$$

This ends the proof.

Finally, instead of considering the complex hyperbolic space form functioning as the underlying manifolds, we can consider the other kind of underlying manifolds such as the Euclidean $n$-space $R^{n}$, the complex $n$-space $C^{n}$ and some conformal flat Riemannian manifolds. Since the corresponding lemmas still exist, one may derive the corresponding estimations over these manifolds. For sake of simplicity, we only state the following.

THEOREM 3.1. Let $\omega$ be a p-form with values in $F$ over $c^{n}$, If the stress energy tensor of $\omega$ is conservative and the norm of $\omega$ over $c^{n}$ is finite or slowly divergent, then $\omega$ vanishes. 
DEFINITION 3.1. A conformal flat Riemannian metric $g$ on $R^{n}$ is called to be of type A if

(A) $g^{2}=f^{2}(x) \sum_{i=1}^{n} x_{1}^{2}$ and the mean curvature normal $H$ of $s_{r}$ never

pointing away from zero. $g$ is called to be of type $B$ if the underlying space is the open unit disc in $R^{n}$ and

$$
\text { (B) } \tilde{\mathrm{g}}=\tilde{\mathrm{f}}^{2}(\mathrm{r}) \sum_{i=1}^{\mathrm{n}} \mathrm{dx} \mathrm{x}_{i}^{2} \text { where } \mathrm{f} \text { satisfying } \int_{0}^{1} \tilde{\mathrm{f}}^{\mathrm{n}-2 \mathrm{p}} \mathrm{dr}=\infty \text { and the mean }
$$

curvature normal $\widetilde{H}$ of $S_{r}$ never pointing away from zero.

THEOREM 3.2. Let $M$ be a conformal flat Riemannian manifold of typed $A$ or $B$. Let $\omega$ be a Riemannian vector bundle valued $p$-form over $M, p>n / 2$, with conservative stress energy tensor. If the norm of $\omega$ over $M$ is finite or slowly divergent. Then $\omega$ vanishes.

ACKNOWLEDGEMENTS. The author would like to thank Professor C.W. Ho and Professor T. Wu for their encouragement and help. He is grateful to the Southern Il1inois University at Edwardsville for its hospitality.

\section{REFERENCES}

1. YAU, S.T. Ha rmonic functions on comlete Riemannian manifolds, comm. Pure Appl. Math., 28 (1975), 201-228.

2. EELLS, J. and SAMPSON, J.H. Ha rmonic mappings of Riemannian manifolds, Amer. J. Math. 86 (1964), 109-160.

3. CHENG, S.Y. Llouville theorem for harmonic maps, Proc. Sym. in Pure Math. 36 (1980), 147-151.

4. GARBER, W.D., RUIJSENAAS, S.H.H., SEILER, E. and BURNS, D. On finite action solutions of the non-1inear $\sigma$-model, Ann. of Physics, 119 (1979), 305-325.

5. HU, H. An nonexistence theorem for harmonic maps with slowly divergent energy, Chin. Ann. of Math., 5B(4) (1984), 737-740.

6. XIN, Y.L. Differential form, conservative law and monolonic formula, Scientia Sinica A 9 (1985).

7. SEALEY, H.C.J. Some conditions ensuring the vanishing of harmonic differential forms with applications to harmonic maps and Yang-Mills theory, Math. Proc. Camb. Ph11. Soc. 91 (1982), 441-452.

8. EELLS, J. and LEMAIRE, L. A report on harmonic maps, Bul1.London Math. Soc. 10 (1978), 1-68.

9. YANO, K. and ISHIHARA, S. Hacmonic and relative affine mappings, J. Diff. Geom. 10 (1975), 501-509.

10. BAIRD, P. and EELLS, J. A conservative 1 aw for harmonic maps, Lecture Notes in Math., 894 Springer Berlin-New York, (1981) 1-25.

11. KOBAYASHI, S. and NOMIZU, K. Foundations of differential geometry, I, II. Interscience, New York, 1963, 1969. 


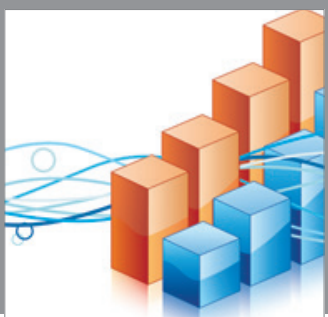

Advances in

Operations Research

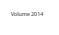

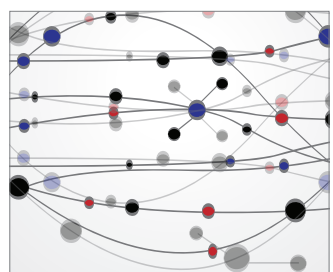

\section{The Scientific} World Journal
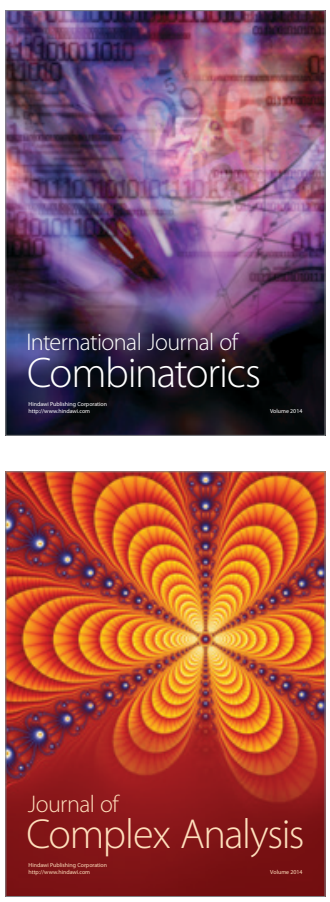

International Journal of

Mathematics and

Mathematical

Sciences
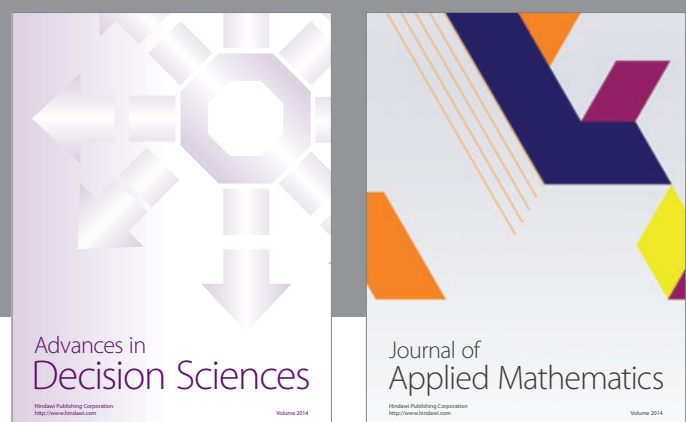

Journal of

Applied Mathematics
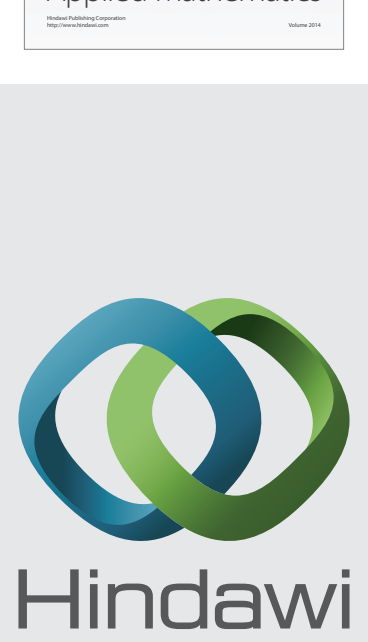

Submit your manuscripts at http://www.hindawi.com
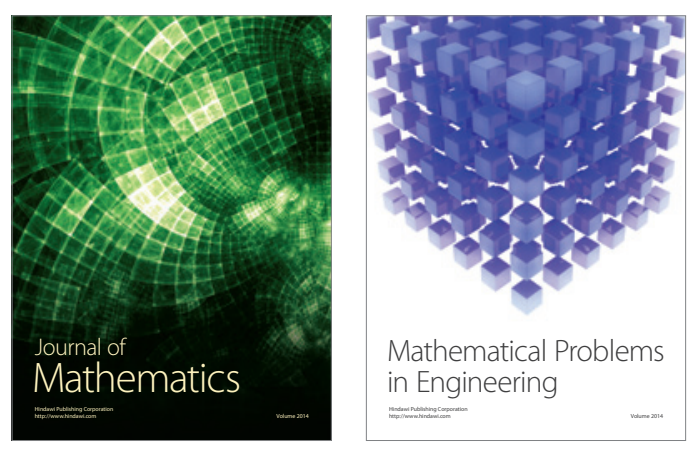

Mathematical Problems in Engineering
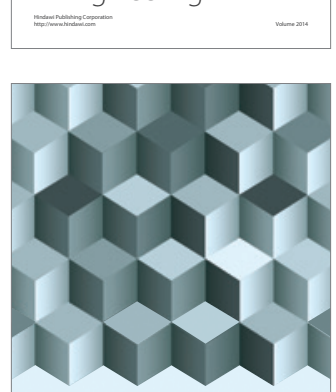

Journal of

Function Spaces
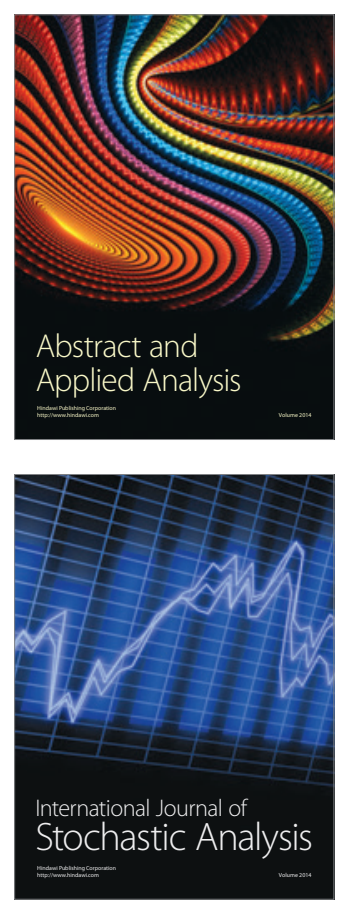

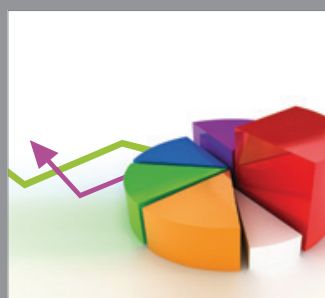

ournal of

Probability and Statistics

Promensencen
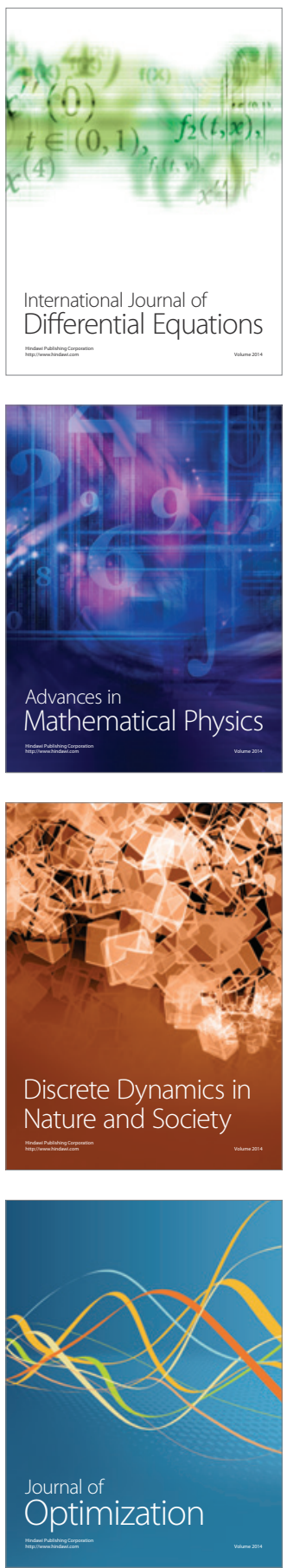